\title{
Modificación del intervalo QT tras el uso de Sevofluorano vs Propofol
}

\author{
Molinari GE. ${ }^{1}$, Molinari GE. ${ }^{1}$ \\ 1 Hospital San Roque, Córdoba, Argentina.
}

Introducción: El intervalo QT en el electrocardiograma puede sufrir una prolongación de origen adquirido, es decir por el uso de numerosos fármacos; o puede tener un origen congénito, debido a la mutación de canales iónicos. El síndrome de QT largo se encuentra asociado a la aparición de pos potenciales tempranos y el aumento de la dispersión del QT en el miocardio. En anestesiología, diariamente se utiliza un gran número de fármacos. La motivación de este trabajo es observar la relación entre el uso de Sevofluorano como fármaco de mantenimiento en anestesia general, y la prolongación del segmento QT, teniendo como base la reconocida capacidad del halogenado de prolongar la repolarización ventricular, comparado con las modificaciones que produce el Propofol.

Métodos: Se realizó un estudio de tipo retrospectivo y analítico en pacientes que se sometieron a procedimientos de baja y moderada complejidad en el Hospital San Roque de la Ciudad de Córdoba, en el período comprendido entre enero y julio del año 2016, se recolectaron datos de las HC de los ECG de los pacientes de cirugías programadas. Medición del segmento QT previo a la exposición de los pacientes a algún tipo de droga, tomando como parámetro electrocardiogramas prequirúrgicos. Posteriormente se formaron dos grupos según la droga utilizada para el mantenimiento anestésico: Sevofluorano o Propofol.

Resultados: La media de QT postquirúrgico, en el caso de los pacientes tratados con Sevofluorano fue de 412,9 mili segundos (D.E $=26,8)$ y en el caso de Propofol fue 420,7 mili segundos (D.E $=22,9)$ la diferencia entre estas medias no fue estadísticamente significativa $(\mathrm{p}>0,05)$.

Conclusión: Todos los pacientes pertenecientes a ambos grupos de estudio sufrieron modificación del intervalo QT post quirúrgico. La media de QT postquirúrgico del grupo de pacientes expuestos a Sevofluorano incremento con respecto a la media de QT prequirúrgico del mismo grupo de pacientes, sin embargo, fue inferior a la media de QT posquirúrgico del grupo expuesto a Propofol.

https://doi.org/10.25237/congresoclasa2019.102 